Supporting Information Materials

\title{
$\mathrm{Au}_{42}:$ An Alternative Icosahedral Golden Fullerene Cage \\ Yi Gao, Xiao Cheng Zeng* \\ Department of Chemistry, University of Nebraska-Lincoln, Lincoln, Nebraska,68588
}

Table S1: Cartesian coordinates of $I_{\mathrm{h}} \mathrm{Au}_{42}$ with BP86/LANL2DZ.

Table S2: Cartesian coordinates of $I_{\mathrm{h}} \mathrm{Au}_{42}$ with B3LYP/LANL2DZ.

Table S3: Harmonic frequencies (IR intensity in parenthesis) of $I_{\mathrm{h}} \mathrm{Au}_{42}$ with BP86/LANL2DZ and B3LYP/LANL2DZ.

Table S4: Electronic energies, entropies, zero-point correction with BP86/LANL2DZ and B3LYP/LANL2DZ.

Reference 17 
Table S1. Cartesian coordinates of $I_{\mathrm{h}} \mathrm{Au}_{42}$ with BP86/LANL2DZ.

\begin{tabular}{|c|c|c|c|}
\hline $\mathrm{Au}$ & -2.78255300 & -3.82985500 & 2.36698100 \\
\hline $\mathrm{Au}$ & 2.78255300 & 3.82985500 & -2.36698100 \\
\hline $\mathrm{Au}$ & 0.00000000 & -4.73396100 & -2.36698100 \\
\hline $\mathrm{Au}$ & 0.00000000 & 4.73396100 & 2.36698100 \\
\hline $\mathrm{Au}$ & 0.00000000 & 0.00000000 & -5.29273000 \\
\hline $\mathrm{Au}$ & 0.00000000 & 0.00000000 & 5.29273000 \\
\hline $\mathrm{Au}$ & 1.43506400 & 1.97519700 & -3.95039400 \\
\hline $\mathrm{Au}$ & -1.43506400 & -1.97519700 & 3.95039400 \\
\hline $\mathrm{Au}$ & 1.43506400 & -1.97519700 & 3.95039400 \\
\hline $\mathrm{Au}$ & -1.43506400 & 1.97519700 & -3.95039400 \\
\hline $\mathrm{Au}$ & 0.00000000 & -3.95039400 & 2.44147800 \\
\hline $\mathrm{Au}$ & 0.00000000 & 3.95039400 & -2.44147800 \\
\hline $\mathrm{Au}$ & 2.78255300 & -3.82985500 & 2.36698100 \\
\hline $\mathrm{Au}$ & -2.78255300 & 3.82985500 & -2.36698100 \\
\hline $\mathrm{Au}$ & 1.43506400 & -4.41667400 & 0.00000000 \\
\hline $\mathrm{Au}$ & -1.43506400 & 4.41667400 & 0.00000000 \\
\hline $\mathrm{Au}$ & -1.43506400 & -4.41667400 & 0.00000000 \\
\hline $\mathrm{Au}$ & 1.43506400 & 4.41667400 & 0.00000000 \\
\hline $\mathrm{Au}$ & 3.75704800 & 2.72965500 & 0.00000000 \\
\hline $\mathrm{Au}$ & -3.75704800 & -2.72965500 & 0.00000000 \\
\hline $\mathrm{Au}$ & 4.50226500 & 1.46287500 & 2.36698100 \\
\hline $\mathrm{Au}$ & -4.50226500 & -1.46287500 & -2.36698100 \\
\hline $\mathrm{Au}$ & 4.64396600 & 0.00000000 & 0.00000000 \\
\hline $\mathrm{Au}$ & -4.64396600 & 0.00000000 & 0.00000000 \\
\hline $\mathrm{Au}$ & 3.75704800 & 1.22073900 & -2.44147800 \\
\hline $\mathrm{Au}$ & -3.75704800 & -1.22073900 & 2.44147800 \\
\hline $\mathrm{Au}$ & 4.50226500 & -1.46287500 & -2.36698100 \\
\hline $\mathrm{Au}$ & -4.50226500 & 1.46287500 & 2.36698100 \\
\hline $\mathrm{Au}$ & 3.75704800 & -2.72965500 & 0.00000000 \\
\hline $\mathrm{Au}$ & -3.75704800 & 2.72965500 & 0.00000000 \\
\hline $\mathrm{Au}$ & 2.32198300 & -3.19593600 & -2.44147800 \\
\hline $\mathrm{Au}$ & -2.32198300 & 3.19593600 & 2.44147800 \\
\hline $\mathrm{Au}$ & 2.32198300 & -0.75445800 & -3.95039400 \\
\hline $\mathrm{Au}$ & -2.32198300 & 0.75445800 & 3.95039400 \\
\hline $\mathrm{Au}$ & -2.32198300 & -0.75445800 & -3.95039400 \\
\hline $\mathrm{Au}$ & 2.32198300 & 0.75445800 & 3.95039400 \\
\hline $\mathrm{Au}$ & 3.75704800 & -1.22073900 & 2.44147800 \\
\hline $\mathrm{Au}$ & -3.75704800 & 1.22073900 & -2.44147800 \\
\hline $\mathrm{Au}$ & -2.32198300 & -3.19593600 & -2.44147800 \\
\hline $\mathrm{Au}$ & 2.32198300 & 3.19593600 & 2.44147800 \\
\hline $\mathrm{Au}$ & 0.00000000 & -2.44147800 & -3.95039400 \\
\hline $\mathrm{Au}$ & 0.00000000 & 2.44147800 & 3.95039400 \\
\hline
\end{tabular}


Table S2. Cartesian coordinates of $I_{\mathrm{h}} \mathrm{Au}_{42}$ with B3LYP/LANL2DZ.

\begin{tabular}{|c|c|c|c|}
\hline $\mathrm{Au}$ & -2.81390700 & -3.87301100 & 2.39365200 \\
\hline $\mathrm{Au}$ & 2.81390700 & 3.87301100 & -2.39365200 \\
\hline $\mathrm{Au}$ & 0.00000000 & -4.78730500 & -2.39365200 \\
\hline $\mathrm{Au}$ & 0.00000000 & 4.78730500 & 2.39365200 \\
\hline $\mathrm{Au}$ & 0.00000000 & 0.00000000 & -5.35236900 \\
\hline $\mathrm{Au}$ & 0.00000000 & 0.00000000 & 5.35236900 \\
\hline $\mathrm{Au}$ & 1.45990000 & 2.00937900 & -4.01875900 \\
\hline $\mathrm{Au}$ & -1.45990000 & -2.00937900 & 4.01875900 \\
\hline $\mathrm{Au}$ & 1.45990000 & -2.00937900 & 4.01875900 \\
\hline $\mathrm{Au}$ & -1.45990000 & 2.00937900 & -4.01875900 \\
\hline $\mathrm{Au}$ & 0.00000000 & -4.01875900 & 2.48373000 \\
\hline $\mathrm{Au}$ & 0.00000000 & 4.01875900 & -2.48373000 \\
\hline $\mathrm{Au}$ & 2.81390700 & -3.87301100 & 2.39365200 \\
\hline $\mathrm{Au}$ & -2.81390700 & 3.87301100 & -2.39365200 \\
\hline $\mathrm{Au}$ & 1.45990000 & -4.49310900 & 0.00000000 \\
\hline $\mathrm{Au}$ & -1.45990000 & 4.49310900 & 0.00000000 \\
\hline $\mathrm{Au}$ & -1.45990000 & -4.49310900 & 0.00000000 \\
\hline $\mathrm{Au}$ & 1.45990000 & 4.49310900 & 0.00000000 \\
\hline $\mathrm{Au}$ & 3.82206700 & 2.77689400 & 0.00000000 \\
\hline $\mathrm{Au}$ & -3.82206700 & -2.77689400 & 0.00000000 \\
\hline $\mathrm{Au}$ & 4.55299700 & 1.47935800 & 2.39365200 \\
\hline $\mathrm{Au}$ & -4.55299700 & -1.47935800 & -2.39365200 \\
\hline $\mathrm{Au}$ & 4.72433400 & 0.00000000 & 0.00000000 \\
\hline $\mathrm{Au}$ & -4.72433400 & 0.00000000 & 0.00000000 \\
\hline $\mathrm{Au}$ & 3.82206700 & 1.24186500 & -2.48373000 \\
\hline $\mathrm{Au}$ & -3.82206700 & -1.24186500 & 2.48373000 \\
\hline $\mathrm{Au}$ & 4.55299700 & -1.47935800 & -2.39365200 \\
\hline $\mathrm{Au}$ & -4.55299700 & 1.47935800 & 2.39365200 \\
\hline $\mathrm{Au}$ & 3.82206700 & -2.77689400 & 0.00000000 \\
\hline $\mathrm{Au}$ & -3.82206700 & 2.77689400 & 0.00000000 \\
\hline $\mathrm{Au}$ & 2.36216700 & -3.25124400 & -2.48373000 \\
\hline $\mathrm{Au}$ & -2.36216700 & 3.25124400 & 2.48373000 \\
\hline $\mathrm{Au}$ & 2.36216700 & -0.76751500 & -4.01875900 \\
\hline $\mathrm{Au}$ & -2.36216700 & 0.76751500 & 4.01875900 \\
\hline $\mathrm{Au}$ & -2.36216700 & -0.76751500 & -4.01875900 \\
\hline $\mathrm{Au}$ & 2.36216700 & 0.76751500 & 4.01875900 \\
\hline $\mathrm{Au}$ & 3.82206700 & -1.24186500 & 2.48373000 \\
\hline $\mathrm{Au}$ & -3.82206700 & 1.24186500 & -2.48373000 \\
\hline $\mathrm{Au}$ & -2.36216700 & -3.25124400 & -2.48373000 \\
\hline $\mathrm{Au}$ & 2.36216700 & 3.25124400 & 2.48373000 \\
\hline $\mathrm{Au}$ & 0.00000000 & -2.48373000 & -4.01875900 \\
\hline $\mathrm{Au}$ & 0.00000000 & 2.48373000 & 4.01875900 \\
\hline
\end{tabular}


Table S3. Harmonic frequencies (IR intensity in parenthesis) of $I_{\mathrm{h}} \mathrm{Au}_{42}$ with BP86/LANL2DZ and B3LYP/LANL2DZ.

\begin{tabular}{|c|c|c|}
\hline & BP86/LANL2DZ & B3LYP/LANL2DZ \\
\hline Frequency $\left(\mathrm{cm}^{-1}\right)$ & 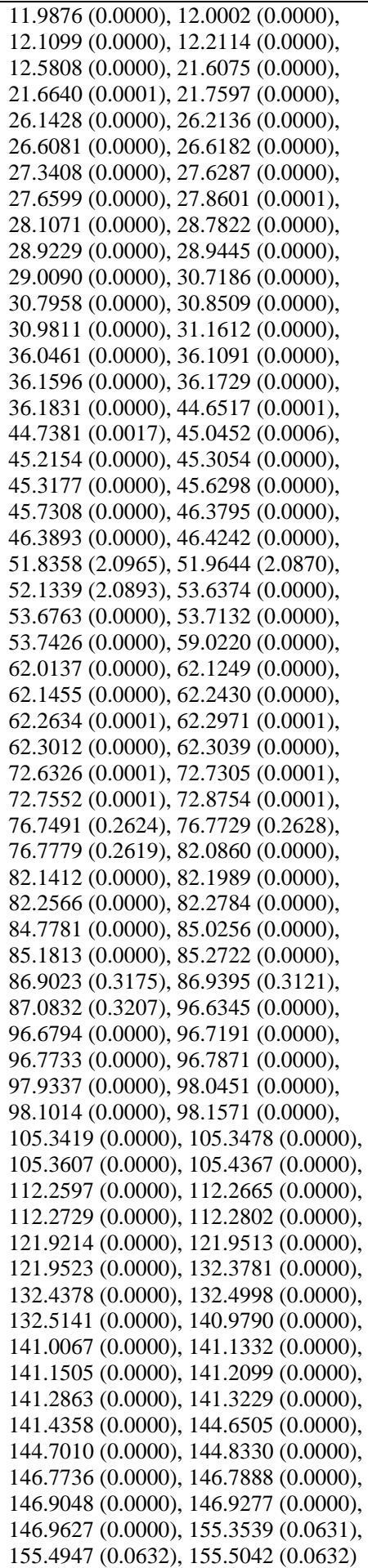 & 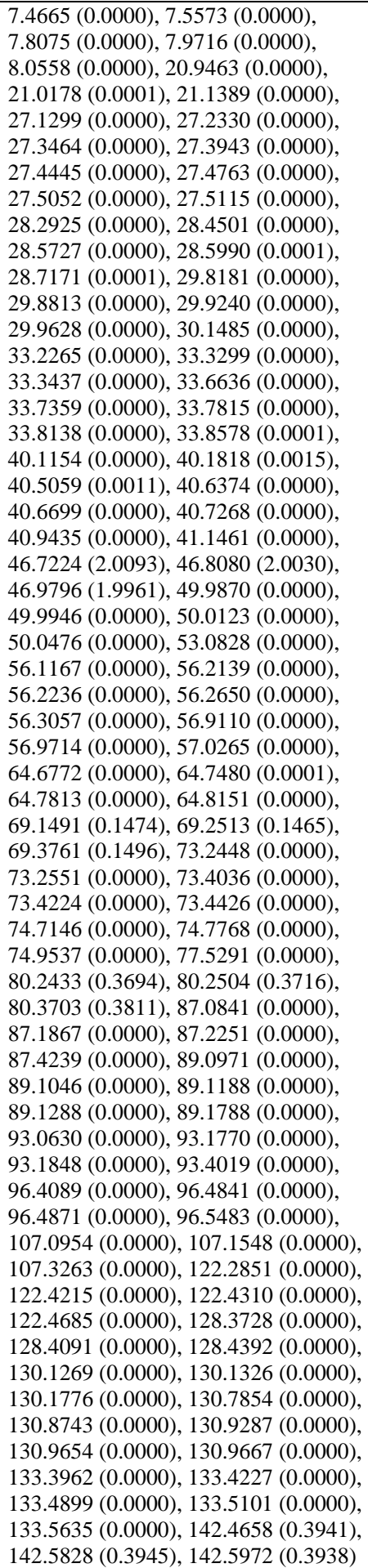 \\
\hline
\end{tabular}


Table S4: Electronic energies, entropies, zero-point correction with BP86/LANL2DZ and B3LYP/LANL2DZ.

\begin{tabular}{lll}
\hline & BP86/LANL2DZ & B3LYP/LANL2DZ \\
\hline Electronic energy (Hartree) & -5694.7699603 & -5691.2776956 \\
Zero-point correction (Hartree) & 0.020934 & 0.018952 \\
Entropy (Cal/Mol-Kelvin) & 622.965 & 647.662 \\
\hline
\end{tabular}

\section{Reference 17:}

Frisch, M. J.; Trucks, G. W.; Schlegel, H. B.; Scuseria, G. E.; Robb, M. A.; Cheeseman, J. R.; Montgomery, Jr., J. A.; Vreven, T.; Kudin, K. N.; Burant, J. C.; Millam, J. M.; Iyengar, S. S.; Tomasi, J.; Barone, V.; Mennucci, B.; Cossi, M.; Scalmani, G.; Rega, N.; Petersson, G. A.; Nakatsuji, H.; Hada, M.; Ehara, M.; Toyota, K.; Fukuda, R.; Hasegawa, J.; Ishida, M.; Nakajima, T.; Honda, Y.; Kitao, O.; Nakai, H.; Klene, M.; Li, X.; Knox, J. E.; Hratchian, H. P.; Cross, J. B.; Bakken, V.; Adamo, C.; Jaramillo, J.; Gomperts, R.; Stratmann, R. E.; Yazyev, O.; Austin, A. J.; Cammi, R.; Pomelli, C.; Ochterski, J. W.; Ayala, P. Y.; Morokuma, K.; Voth, G. A.; Salvador, P.; Dannenberg, J. J.; Zakrzewski, V. G.; Dapprich, S.; Daniels, A. D.; Strain, M. C.; Farkas, O.; Malick, D. K.; Rabuck, A. D.; Raghavachari, K.; Foresman, J. B.; Ortiz, J. V.; Cui, Q.; Baboul, A. G.; Clifford, S.; Cioslowski, J.; Stefanov, B. B.; Liu, G.; Liashenko, A.; Piskorz, P.; Komaromi, I.; Martin, R. L.; Fox, D. J.; Keith, T.; Al-Laham, M. A.; Peng, C. Y.; Nanayakkara, A.; Challacombe, M.; Gill, P. M. W.; Johnson, B.; Chen, W.; Wong, M. W.; Gonzalez, C.; and Pople, J. A. Gaussian 03, Revision C.02, Gaussian, Inc., Wallingford CT, 2004. 